Mens

revue d'histoire intellectuelle de l'Amérique française

Philip Massolin. Canadian Intellectuals, the Tory Tradition, and the Challenge of Modernity, 1939-1970. Toronto, University of Toronto Press, 2001. x-357 p.

\title{
Damien-Claude Bélanger
}

Volume 3, numéro 2, printemps 2003

URI : https://id.erudit.org/iderudit/1024649ar

DOI : https://doi.org/10.7202/1024649ar

Aller au sommaire du numéro

Éditeur(s)

Centre de recherche en civilisation canadienne-française

ISSN

1492-8647 (imprimé)

1927-9299 (numérique)

Découvrir la revue

Citer ce compte rendu

Bélanger, D.-C. (2003). Compte rendu de [Philip Massolin. Canadian Intellectuals, the Tory Tradition, and the Challenge of Modernity, 1939-1970.

Toronto, University of Toronto Press, 2001. x-357 p.] Mens, 3(2), 288-294.

https://doi.org/10.7202/1024649ar d'utilisation que vous pouvez consulter en ligne.

https://apropos.erudit.org/fr/usagers/politique-dutilisation/ 
une règle générale selon laquelle l'ethnicité se transmettait par le père. Cette règle était toutefois amendée en ce qui concernait les Canadiens français : était statistiquement d'origine française celui ou celle dont un des deux parents était né au Québec. Taché a ainsi fait des Canadiens français le groupe ethnique le plus important au Canada non pas en truquant les résultats ou en modifiant les chiffres obtenus lors du recensement, mais en définissant les termes et en posant les questions de manière à faire ressortir, si ce n'est à augmenter artificiellement, l'importance de son propre groupe ethnique.

En définitive, cet ouvrage historique mérite tous les honneurs qu'il a reçus (prix Sir John A. Macdonald de la Société historique du Canada et le prix John Porter de la Société canadienne de sociologie et d'anthropologie). Clair, nuancé, intelligent, original, pertinent, il apporte à l'histoire du $\mathrm{XIX}^{\mathrm{e}}$ siècle une contribution précieuse et exemplaire à l'étude d'une source incontournable en elle-même et du développement de l'État canadien en général.

Michel Ducharme

Département d'histoire, Université MoGill

\section{Philip Massolin. Canadian Intellectuals, the Tory Tradition, and the Challenge of Modernity, 1939- 1970. Toronto, University of Toronto Press, 2001. x- $357 \mathrm{p}$.}

Inégaux, répétitifs ou incomplets, certains ouvrages peuvent néanmoins transcender leurs lacunes fondamentales, car parfois la compétence compense largement l'insuffisance. Canadian Intellectuals, the Tory Tradition, and the Challenge of Modernity est un de ces livres. Paru en 2001, il a malheureuse- 
ment été plus ou moins ignoré par la critique, peut-être parce que son auteur, Philip Massolin, n'œuvre pas dans le milieu universitaire. Diplômé de l'Université de l'Alberta - cet ouvrage est d'ailleurs une version remaniée de sa thèse de doctorat préparée sous la direction du très respecté professeur Doug Owram, - l'auteur est présentement directeur de la recherche chez Chinook Multimedia, une entreprise albertaine spécialisée dans le développement de matériel pédagogique historique canadien.

L'étude de Massolin est centrée sur l'antimodernisme de huit intellectuels anglo-canadiens que l'auteur situe dans la tradition intellectuelle conservatrice : Harold Innis, Donald Creighton, Vincent Massey, Hilda Neatby, George Parkin Grant, William L. Morton, Northrop Frye et Marshall McLuhan. Objet d'étude pourtant fuyant et compliqué, la modernité est abordée dans ce livre avec sagesse et simplicité :

In its most general sense, modernity was the replacement of Victorian society - agrarian, religious, adhering to a rigid set of philosophical and moral codes - with the modern age: industrial, secular, and anti-philosophical. From an economic standpoint, it pertained to the arrival of an urban and industrial society that replaced a hoary agrarian-merchant system. Closely related to the process of urbanindustrialization, modernization also involved the rise of a consumer, scientific-materialist, and technological society. As Canadian society became secularized and the agrarian way of life was eroded, educational systems also became modernized. In many ways, academic modernization is central to the genesis of the modern era. (p. 3)

La conception de la modernité qu'on retrouve dans cet ouvrage est solide, mais un peu trop axée sur l'éducation, sans doute parce que l'auteur tient à examiner la pensée de huit universi- 
taires dont l'unité idéologique provient surtout de «common, historically based concerns » (p. 13), en particulier d'une critique virulente de la modernité pédagogique.

Massolin a parfaitement saisi le pluralisme fondamental de la pensée conservatrice. En effet, le conservatisme est une idéologie plus ou moins allergique à la rigidité doctrinale et qui ne se laisse pas saisir facilement. Ainsi, la définition qu'offre l'auteur des " overarching values of Canadian conservatism " est simple et efficace. Si les intellectuels analysés dans cet ouvrage sont quelque peu divisés quant au rapport à l'Empire britannique, ils partagent néanmoins certaines valeurs communes : "communitarianism, elitism, and an appreciation of organic, evolutionary change » (p. 5).

Malheureusement, l'auteur est moins efficace quand il aborde l'intellectuel en tant qu'objet d'étude. En effet, cet ouvrage ne comprend aucune définition de l'intellectuel. Pourtant, s'il y a une définition qui ne va pas de soi en histoire des idées, c'est bien celle de l'intellectuel! Cette absence de précision est d'autant plus incompréhensible quand on apprend à la page cinquante-quatre que Vincent Massey est en fait un « quasiintellectual ». Dans cet ouvrage, « intellectual » et « academic » sont utilisés de façon à peu près interchangeable.

Les trois premiers chapitres de cette étude sont consacrés à la critique conservatrice de la science et de l'éducation. Massolin y explore l'analyse pénétrante de McLuhan, Innis et Grant des effets déshumanisants de la technologie moderne avant d'aborder la seule critique que partagent tous les intellectuels issus de la " tory tradition » : celle de l'éducation moderne, c'est-à-dire laïque, spécialisée et démocratique. En effet, l'éducation de masse est la bête noire par excellence des conservateurs canadiens-anglais de l'après-guerre. Partisans de l'enseignement classique, ils dénoncent farouchement l'enseignement utilitaire et pragmatique. L'analyse de l'auteur est so- 
lide, mais beaucoup trop détaillée et répétitive. D’ailleurs, il revient constamment au refus conservateur de l'éducation de masse tout au long de cette étude, au point où le lecteur se demande si l'ouvrage n'aurait pas dû plutôt s'intituler Canadian Intellectuals, the Tory Tradition, and the Challenge of Modern Education.

Massolin examine ensuite la critique conservatrice de la culture de masse. Ce chapitre est excellent. L'auteur y offre d'ailleurs une lecture originale du rapport de la Commission Massey-Lévesque, qu'il situe dans la «tory tradition». Pour l'auteur, le combat antiaméricain de Vincent Massey est en fait un combat contre la culture de masse. Antimatérialistes et traditionalistes, plusieurs des intellectuels conservateurs analysés par Massolin s'acharnent contre la culture américaine, car

Since American culture was quickly becoming indistinguishable from mass culture, cultural Americanization implied a homogenous, mass civilization devoid of distinctiveness and connections to the cultural past. American culture, therefore, hindered the critical effort to maintain traditions as a means of building a strong civilization. Along with mass culture and the mass media to which it had been inextricably bound, it presented serious threats to Canada's cultural future. (p. 184)

L'auteur a donc compris les véritables fondements de l'antiaméricanisme de droite. Exacerbé par la politique étrangère des États-Unis, ce phénomène, qu'il soit canadien ou étranger, procède d'abord d'une lecture antilibérale et antimoderne de l'évolution historique.

C'est dans les cinquante dernières pages que l'auteur nous livre la partie la plus consistante et intéressante de son étude. Il se penche alors sur l'évolution et le déclin de la «tory tradition » au Canada anglais. Battu en brèche dans les années soixante, le conservatisme intellectuel canadien-anglais dispa- 
rait complètement avec la mort de Creighton en 1979. Au plan politique, ce courant cesse donc d'exercer une influence quelconque sur le Parti conservateur. En effet, si la pensée politique de Diefenbaker avait été influencée par la «tory tradition ", celle de Clark et de Mulroney ne le sera pas. Au fond, le néoconservatisme intellectuel des années quatre-vingts et quatrevingt-dix n'est qu'un libéralisme de droite pour Massolin. Cependant - et c'est là une des sections les plus intéressantes de cette étude, - certaines des idées de la droite intellectuelle sont récupérées par la «new left» du Canada anglais, notamment son antiaméricanisme et son analyse pénétrante du caractère marginal de l'économie canadienne. Pays né d'un attachement profond à la continuité et à la tradition, le Canada serait une anti-Amérique, voire l'incarnation nationale de la contre-révolution pour Grant et Creighton. Cette interprétation conservatrice est récupérée et déformée par le politologue de gauche Gad Horowitz dans les années soixante. Celuici, s'appuyant sur la thèse des "fragments » de Louis Hartz, prétend que le caractère conservateur de la culture politique du Canada traditionnel favorise l'implantation du socialisme en sol canadien parce que ces deux idéologies partagent un même refus viscéral du libéralisme et de l'individualisme. Au niveau de la culture politique, il serait donc plus facile de passer du conservatisme au socialisme que du libéralisme au socialisme. Ainsi, le Canada aurait de bien meilleures chances d'accéder au socialisme que l'Amérique, qui est une société essentiellement libérale.

Pessimiste, Massolin constate tristement que la « tory tradition » ne subsiste que dans les écrits de quelques gauchistes dépassés ${ }^{1}$. Il a tort. Le traditionalisme conservateur reste présent dans la vie intellectuelle du Canada anglais. Marginal, ce courant se retrouve néanmoins chez certains intellectuels, dont l'historien Desmond Morton, ce « red tory » qui pourfend Tru- 
deau et le Parti libéral, et qui se réclame fièrement de la tradition creightonienne. De plus, le royalisme de la «tory tradition» reste bien vivant au Canada anglais. Les récentes célébrations du jubilé royal ont d'ailleurs révélé que l'attachement d'un important nombre de Canadiens anglais pour la monarchie est réel.

C'est dans l'épilogue qu'apparaît le Québec. Massolin y analyse l'opposition de Creighton et de W.L. Morton au bilinguisme et à la thèse des deux nations. Ces quelques pages laisseront le lecteur Canadien français sur sa faim. En effet, dans cet ouvrage, "Canadian» est malheureusement synonyme d'« English Canadian » et le Québec n'apparaît que très rarement. Au fond, l'auteur n'y est pour rien. Il ne fait que suivre la déplorable évolution générale de l'historiographie canadienne qui tend à faire du Québec et du Canada anglais des entités historiques essentiellement séparées. Nourris par le refus de la canadianité des historiens québécois, ceux du Canada anglais trouvent ainsi une justification commode pour exclure le Québec de leurs études. Le résultat : deux histoires nationales tronquées.

Enfin, soulignons l'oubli le plus important de cet ouvrage : il ne contient à peu près rien sur la critique sociale des intellectuels conservateurs. Pour maintenir le focus de son analyse sur la culture et l'éducation et sans doute pour préserver la cohérence intellectuelle de son groupe d'auteurs, Massolin évacue la famille, l'ethnicité et l'immigration de son étude. Il justifie d'ailleurs ce choix de façon malaisée :

There are other, more marginal aspects [of modernity] that are not dealt with in depth here. This work is less articulate on criticisms of issues such as immigration, ethnicity, and family. The reason is not that these issues are unimportant to the modernity question, but rather that the critics themselves tended to be less 
concerned with them, especially in the light of the more pressing questions. Indeed, the anti-modernists tended to be at best haphazard or at worst inarticulate about these subjects. For some, immigration and the changing ethnic composition of the country did not even qualify as significant aspects of modernity. (pp. 13-14)

Dans l'ensemble, Philip Massolin nous livre une étude de qualité qui révèle la "tory tradition » avec discernement. Ainsi, et en dépit de ses nombreuses faiblesses, Canadian Intellectuals, the Tory Tradition, and the Challenge of Modernity, 19391970 est un ouvrage remarquable et original qui mériterait une diffusion plus importante.

\author{
Damien-Claude Bélanger \\ Département d'bistoire, Université MoGill
}

\title{
NOTES
}

${ }^{1}$ Selon Massolin, la «new left» du Canada anglais serait elle-même moribonde. 